\title{
Calcium Elevation in Astrocytes Causes an NMDA Receptor- Dependent Increase in the Frequency of Miniature Synaptic Currents in Cultured Hippocampal Neurons
}

\author{
Alfonso Araque, Rita P. Sanzgiri, Vladimir Parpura, and Philip G. Haydon \\ Laboratory of Cellular Signaling, Department of Zoology and Genetics, lowa State University, Ames, lowa 50011
}

Astrocytes exhibit a form of excitability and communication on the basis of intracellular $\mathrm{Ca}^{2+}$ variations (Cornell-Bell et al., 1990; Charles et al., 1991) that can be initiated by neuronal activity (Dani et al., 1992; Porter and McCarthy, 1996). A Ca ${ }^{2+}$ elevation in astrocytes induces the release of glutamate (Parpura et al., 1994; Pasti et al., 1997; Araque et al., 1998; Bezzi et al., 1998), which evokes a slow inward current in neurons and modulates action potential-evoked synaptic transmission between cultured hippocampal cells (Araque et al., 1998), suggesting that astrocytes and neurons may function as a network with bidirectional communication. Here we show that a $\mathrm{Ca}^{2+}$ elevation in astrocytes increases the frequency of excitatory as well as inhibitory miniature postsynaptic currents (mPSCs), without modifying their amplitudes. Thapsigargin incubation, microinjection of the $\mathrm{Ca}^{2+}$ chelator BAPTA, and photolysis of the $\mathrm{Ca}^{2+}$ cage NP-EGTA demonstrate that $\mathrm{a} \mathrm{Ca}^{2+}$ elevation in astrocytes is both necessary and sufficient to modulate spontaneous transmitter release. This $\mathrm{Ca}^{2+}$-dependent release of glutamate from astrocytes enhances mPSC frequency by acting on NMDA glutamate receptors, because it is antagonized by D-2-amino-5-phosphonopentanoic acid (AP5) or extracellular $\mathrm{Mg}^{2+}$. These NMDA receptors are located extrasynaptically, because blockage specifically of synaptic NMDA receptors by synaptic activation in the presence of the open channel blocker MK-801 did not impair the AP5-sensitive astrocyte-induced increase of $\mathrm{mPSC}$ frequency. Therefore, astrocytes modulate spontaneous excitatory and inhibitory synaptic transmission by increasing the probability of transmitter release via the activation of NMDA receptors.

Key words: astrocyte-neuron signaling; NMDA glutamate receptors; calcium cage photolysis; calcium waves; miniature synaptic currents; hippocampal synaptic transmission
There is growing evidence indicating that astrocytes may play more active roles in the CNS beyond the simple structural and trophic support for neurons. The existence of signaling between astrocytes, using intracellular $\mathrm{Ca}^{2+}$ variations that can propagate as intercellular $\mathrm{Ca}^{2+}$ waves (Cornell-Bell et al., 1990; Charles et al., 1991; Finkbeiner, 1992; Newman and Zahs, 1997), the susceptibility of astrocytes to respond to neuronal activity (Dani et al., 1992; Porter and MacCarthy, 1996) by the activation of neurotransmitter receptors that trigger $\mathrm{Ca}^{2+}$ waves (Cornell-Bell et al., 1990; Charles et al., 1991; Murphy et al., 1993; Duffy and MacVicar, 1995), and the ability of astrocytes to signal to neurons by releasing glutamate that induces a neuronal $\mathrm{Ca}^{2+}$ elevation (Charles, 1994; Nedergaard, 1994; Parpura et al., 1994; Hassinger et al., 1995; Pasti et al., 1997; Araque et al., 1998; Bezzi et al., 1998) suggest that astrocytes and neurons may function as a network in which bidirectional communication takes place.

Although the existence of communication between astrocytes and neurons is firmly established by $\mathrm{Ca}^{2+}$ imaging studies (Charles, 1994; Nedergaard, 1994; Parpura et al., 1994; Hassinger et al., 1995; Pasti et al., 1997; Bezzi et al., 1998), the physiological

\footnotetext{
Received April 13, 1998; revised June 11, 1998; accepted June 22, 1998.

This work was supported by Grants from National Institutes of Health (NS24233 and NS37585) and the Iowa State University Biotechnology Council to P.G.H. and by a long-term postdoctoral fellowship from the Human Frontier Science Program to A.A. We thank Prairie Technologies (Waunakee, WI) for a gift of UVtransmitting optical fiber and for the proprietary knowledge used to construct our fiber photolysis unit.

Correspondence should be addressed to Dr. Philip G. Haydon, Laboratory of Cellular Signaling, Department of Zoology and Genetics, Room 339 Science II, Iowa State University, Ames, IA 50011. E-mail: pghaydon@iastate.edu Copyright (C) 1998 Society for Neuroscience $0270-6474 / 98 / 186822-08 \$ 05.00 / 0$
}

consequences of such communication have not been elucidated. However, we have shown recently that the electrical or mechanical stimulation of astrocytes evokes in adjacent neurons a glutamate-dependent slow inward current mediated by the activation of both NMDA and non-NMDA glutamate receptors (Araque et al., 1998). We also have provided evidence for the involvement of astrocytes in the modulation of action potentialevoked synaptic transmission via the activation of presynaptic metabotropic glutamate receptors (mGluRs). In addition to these electrophysiological consequences of the astrocyte-neuron signal, we also have reported that astrocyte stimulation may increase the frequency of miniature postsynaptic currents (mPSCs) via undefined mechanisms. Therefore, the aim of the present work was to characterize the mechanisms underlying this astrocyteinduced modulation of mPSC frequency.

We used three different stimuli-mechanical, electrical, or UV photolysis of a $\mathrm{Ca}^{2+}$ cage - to raise the intracellular $\mathrm{Ca}^{2+}$ concentration $\left(\left[\mathrm{Ca}^{2+}\right]_{\mathrm{i}}\right)$ in astrocytes while mPSCs were recorded in adjacent neurons. We found that astrocyte stimulation transiently increased the frequency of excitatory as well as inhibitory mPSCs, without modifying their amplitude distribution. The elevation of intracellular $\mathrm{Ca}^{2+}$ in astrocytes was both necessary and sufficient to induce an increase in mPSC frequency and was prevented by the NMDA receptor antagonist D-2-amino-5-phosphonopentanoic acid (AP5), indicating that it was mediated by the activation of NMDA receptors. Because the astrocyte-induced increase in mPSC frequency was not impaired after the selective block of synaptic NMDA receptors with the use-dependent open-channel blocker MK-801, we conclude that astrocytes can increase the 
frequency of mPSCs by activating extrasynaptic NMDA receptors.

\section{MATERIALS AND METHODS}

Culture preparation. Primary cultures of mixed hippocampal neurons and astrocytes from 1- to 3-d-old postnatal rats were prepared as previously described (Araque et al., 1998) and were used after 8-25 d in culture, at a time when synapses were established. At the time of use, astrocytes were confluent in these cultures.

Electrophysiology. Whole-cell patch-clamp recordings were obtained from neurons with an Axopatch-1C amplifier and pClamp software (Axon Instruments, Foster City, CA). Currents were filtered at $1-2 \mathrm{kHz}$ and sampled above $2 \mathrm{kHz}$. External control solution contained (in $\mathrm{mm}$ ): $140 \mathrm{NaCl}, 5 \mathrm{KCl}, 4 \mathrm{CaCl}_{2}, 10 \mathrm{HEPES}, 10$ glucose, and 6 sucrose, $\mathrm{pH}$ 7.35. mPSCs were recorded in $1 \mu \mathrm{M}$ tetrodotoxin (TTX). To optimize NMDA receptor activation, we omitted $\mathrm{Mg}^{2+}$ and added $10 \mu \mathrm{M}$ glycine to the solution. High osmolarity solution was obtained by adding $0.3 \mathrm{M}$ sucrose. In $4 \mathrm{~mm} \mathrm{Mg}{ }^{2+}$ solution, $\mathrm{MgCl}_{2}$ was added without osmolarity compensation. The patch pipette solution contained (in $\mathrm{mM}$ ): 140 K-gluconate, 10 EGTA, 4 Mg-ATP, 0.2 Tris-GTP, and 10 HEPES, $\mathrm{pH}$ 7.35. In some experiments, $10 \mathrm{~mm}$ BAPTA (1,2-bis(2-aminophenoxy) ethane- $N, N, N^{\prime}, N^{\prime}$-tetraacetic acid; tetrapotassium salt) was added to the pipette solution. Unless stated otherwise, the membrane potential was held at $-60 \mathrm{mV}$ to study miniature EPSCs (mEPSCs), at $-10 \mathrm{mV}$ to analyze miniature IPSCs (mIPSCs), or at $-30 \mathrm{mV}$ to permit simultaneous observation of both mEPSCs and mIPSCs. At the holding potentials that were used, glutamatergic EPSCs and GABAergic IPSCs were identified as inward and outward currents, respectively (Araque et al., 1998). For thapsigargin treatment the cells were incubated with $1 \mu \mathrm{M}$ thapsigargin for 30-60 min. In some experiments, synaptic transmitter release was increased by using high osmolarity solution $(0.3 \mathrm{M}$ sucrose was added to the control solution) delivered from a micropipette (tip diameter, $\sim 2 \mu \mathrm{m}$ ) by 1 - to 50 -sec-duration pressure pulses (Picospritzer II, General Valve, Fairfield, NJ).

The morphological identification of neurons was confirmed electrophysiologically by their ability to generate TTX-sensitive $\mathrm{Na}^{+}$-mediated action potentials and by the presence of fast synaptic currents. Confluent astrocytes, $\sim 25-150 \mu \mathrm{m}$ from the soma of the recorded neuron, were stimulated mechanically via glass micropipettes filled with external saline (see Charles, 1994; Nedergaard, 1994; Araque et al., 1998). Similar results were obtained when voltage pulses ( $1 \mathrm{msec}$ duration, $150 \mathrm{~V}$ ) were used to stimulate astrocytes (see Araque et al., 1998). However, to prevent the potential effects of direct electrical stimulation on synaptic terminals, the results presented were obtained mainly by using mechanical stimulation, unless stated otherwise. At least eight astrocytes were stimulated in each parallel control, and test conditions and data were obtained from at least three different experiments (i.e., at least 24 astrocytes were stimulated in each condition). The incidence of astrocyte-induced responses was defined as the proportion of responses relative to the total number of astrocytes stimulated in each experiment; statistical differences were established with the Student's $t$ test. All experiments were performed at room temperature $\left(20-23^{\circ} \mathrm{C}\right)$. Data are expressed as mean \pm SEM.

Analysis of mPSCs was done by using the ACSPLOUF software (obtained from Dr. Pierre Vincent, University of California at San Diego). The cumulative probabilities of the mPSC amplitude and frequency before and after astrocyte stimulation were plotted. The mPSC frequency was calculated in $1 \mathrm{sec}$ bins. Cumulative probability plots 15-30 sec before and after astrocyte stimulation were compared, and significant differences were established at $p<0.05$, using the Kolmogorov-Smirnov test.

Neuronal labeling with tetanus toxin. Neurons were identified by labeling with the fluorescein-conjugated $\mathrm{C}$-fragment of tetanus toxin $(\mathrm{C}$ FITC, List Biological Laboratories, Campbell, CA) by a modification of a previously described procedure (Charles, 1994), as reported elsewhere (Araque et al., 1998). Cells were incubated for $1 \mathrm{hr}$ in $10 \mu \mathrm{g} / \mathrm{ml}$ of $\mathrm{C}$-FITC at $37^{\circ} \mathrm{C}$. After a wash period the cells were viewed with fluorescein optics.

Calcium measurements. The ability of stimuli to evoke a wave of elevated $\mathrm{Ca}^{2+}$ in astrocytes was monitored by fluorescence microscopy with the $\mathrm{Ca}^{2+}$ indicator fluo-3. Cultures were incubated at $37^{\circ} \mathrm{C}$ for 45 min with the acetoxymethyl ester of fluo-3 (fluo-3-AM, $10 \mu \mathrm{g} / \mathrm{ml}$; Molecular Probes, Eugene, OR). After being washed, the indicator was allowed to deesterify for $45 \mathrm{~min}$. Coverslips containing fluo-3-loaded cells were visualized via a silicon intensified target (SIT) camera
(Hamamatsu Photonic System, Bridgewater, NJ) or IC-300 intensified charge-coupled device (CCD) camera (Photon Technology, Monmouth Junction, NJ) attached to a Nikon 300 inverted microscope and a $\mathrm{NeD}_{\mathrm{LC}}$ optical workstation (Prairie Technologies, LLC, Waunakee, WI). Quantitative fluorescence measurements were made with the $\mathrm{NeD}_{\mathrm{LC}}$ video software.

Microinjection into astrocytes. In some experiments we injected the $\mathrm{Ca}^{2+}$ chelator BAPTA into individual astrocytes, as described elsewhere (Araque et al., 1998). Microinjection pipettes (tip diameter, $\sim 400 \mathrm{~nm}$ ) were pulled from Kwik-Fil borosilicate glass capillaries (World Precision Instruments, Sarasota, FL), using a Sutter P-2000 micropipette puller (Sutter Instrument, Novato, CA). Pipettes were filled with a solution containing $375 \mathrm{~mm}$ BAPTA, pH 7.2, and $0.25 \mathrm{~mm}$ fluoro-ruby. This solution was pressure-injected into single astrocytes, using a $300 \mathrm{msec}, 15$ psi pulse (Narishige IM-200, Narishige, Greenvale, NY). The positioning of micropipettes was controlled by an Eppendorf micromanipulator. Based on quantification of the fluoro-ruby fluorescence, the resulting final intracellular BAPTA concentration was estimated to be $1-2 \mathrm{~mm}$ (Araque et al., 1998). In other experiments, NP-EGTA (o-nitrophenylEGTA, tetrapotassium salt; Molecular Probes, Eugene, OR) was injected into individual astrocytes from pipettes filled with $15 \mathrm{~mm}$ NP-EGTA and $0.25 \mathrm{~mm}$ fluoro-ruby.

\section{RESULTS}

We have demonstrated previously that stimuli that elevate $\left[\mathrm{Ca}^{2+}\right]_{\mathrm{i}}$ in astrocytes can induce an increase in the frequency of mPSCs in adjacent neurons (Araque et al., 1998). To evaluate further the mechanism responsible for this response, we mechanically stimulated astrocytes while miniature synaptic currents were recorded from adjacent neurons. The frequency of both mEPSCs and mIPSCs was increased by mechanical stimulation of the astrocytes (Figs. 1, 2). The mEPSCs frequency was increased after stimulation of $48.6 \pm 3.8 \%$ of the astrocytes $(n=20)$. In those cells that responded to astrocyte stimulation, mEPSC frequency increased, reaching a maximum (mean maximum increase was $12.2 \pm 2.6$ times the control frequency; $n=21$ ) and declined slowly, usually lasting for $\sim 1-2 \mathrm{~min}$ before returning to prestimulus values (Fig. $1 B$ ). This increase in the frequency of miniature synaptic currents was also obvious by comparing the cumulative probability plots of the mEPSC frequency $30 \mathrm{sec}$ before and after astrocyte stimulation (Fig. 1C, open and filled symbols, respectively; $p<0.005)$. This increased mEPSC frequency was not accompanied by perceptible changes in the profile of the histograms of the mEPSC amplitudes (Fig. $1 D, E$ ) nor by statistically significant changes in the corresponding cumulative probability plots (Fig. $1 F, G)$. Likewise, mIPSC frequency increase was elicited by $37.2 \pm 3.7 \%$ of stimulated astrocytes $(n=$ $26)$, the mean maximum increase in MIPSC frequency was $11.7 \pm$ 2.3 times the prestimulus level $(n=22)$, and it showed a similar time course to the change in mEPSC frequency (Fig. $2 A-C$ ). Moreover, astrocytes increased the frequency of mIPSCs without modifying their amplitude (Fig. 2D-G). Taken together, these data suggest that stimulation of the astrocyte caused an increase in the probability of presynaptic transmitter release from both excitatory and inhibitory neurons.

\section{Elevation of $\mathrm{Ca}^{2+}$ in astrocytes is both necessary and sufficient to modulate synaptic transmission}

To control for the possibility that our mechanical stimuli directly activate neuronal processes, we used the FITC-labeled C-fragment of tetanus toxin, which selectively labels the soma and neurites of the neurons, but not astrocytes, thus allowing for the visualization of neuronal processes in living preparations and permitting the selective stimulation of neurite-free regions of astrocytes (Araque et al., 1998). When we used this method to disclose neuronal processes, specific stimulation of astrocytes still 
A
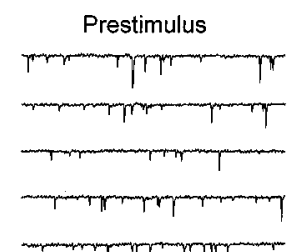

$\mathrm{B}$

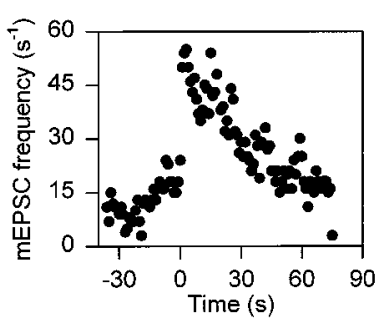

$\mathrm{D}$

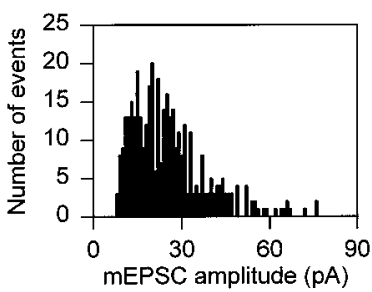

$\mathrm{F}$

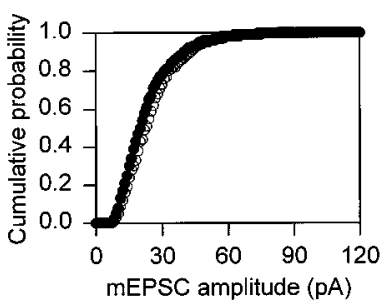

Poststimulus

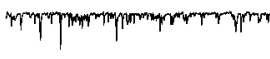

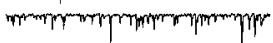

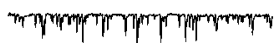

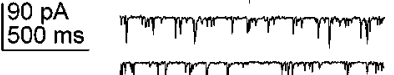

C

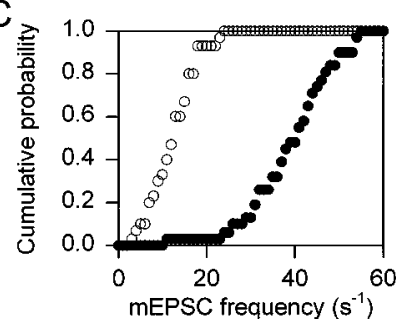

E

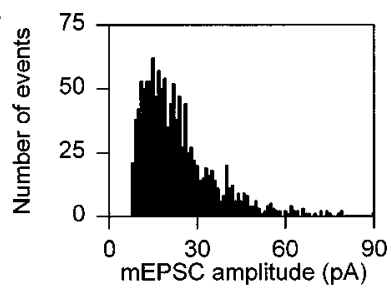

$\mathrm{G}$

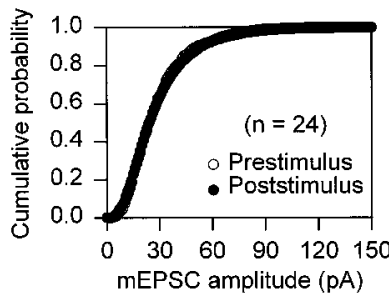

Figure 1. Astrocyte stimulation increases mEPSC frequency. $A$, mEPSCs recorded at a holding potential of $-60 \mathrm{mV}$ before and after mechanical stimulation of an astrocyte. $B$, Time course of the mEPSC frequency calculated in $1 \mathrm{sec}$ bins. Zero time corresponds to the time of astrocyte stimulation. $C$, Cumulative probability plot of the mEPSC frequency $30 \mathrm{sec}$ before and after astrocyte stimulation (open and filled symbols, respectively). $D, E$, Histograms of mEPSC amplitudes (bin width, $1 \mathrm{pA}$ ) recorded 30 sec before and after astrocyte stimulation, respectively. $F$, Cumulative probability plot of the mEPSC amplitudes recorded $30 \mathrm{sec}$ before and after astrocyte stimulation (open and filled symbols, respectively). $G$, Average $(n=24)$ cumulative probability plot of the mEPSC amplitudes recorded 30 sec before and after astrocyte stimulation (open and filled symbols, respectively). Error bars showing SEM are smaller than symbol size. To obtain cumulative probability plots, we calculated the frequency and the amplitudes of mEPSCs in $1 \mathrm{sec}$ and $1 \mathrm{pA}$ bins, respectively.

evoked an increase in mPSC frequency (13 of 24 astrocytes). Although we cannot discount the possibility that unlabeled processes were still present in these cultures, these results suggest that astrocytes are responsible for increasing the mPSC frequency.

We have demonstrated previously that an elevation of $\mathrm{Ca}^{2+}$ in astrocytes is necessary to evoke astrocyte-induced slow inward current in adjacent neurons (Araque et al., 1998). Therefore, we asked whether a $\mathrm{Ca}^{2+}$ elevation in these cells was also necessary for the increase in mPSC frequency. Astrocyte $\mathrm{Ca}^{2+}$ waves require functional internal $\mathrm{Ca}^{2+}$ stores that can be depleted with the $\mathrm{Ca}^{2+}$-ATPase inhibitor thapsigargin (Charles et al., 1993; Newman and Zahs, 1997; Araque et al., 1998). After thapsigargin incubation the mechanical stimulation of astrocytes no longer led to a $\mathrm{Ca}^{2+}$ wave (data not shown; but see Araque et al., 1998), and

A

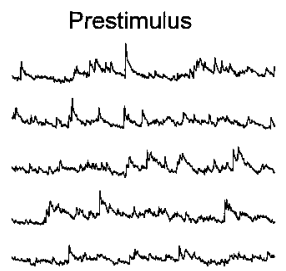

B

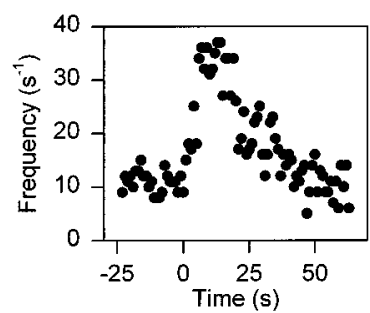

D

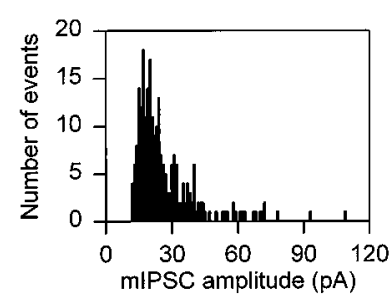

$E$
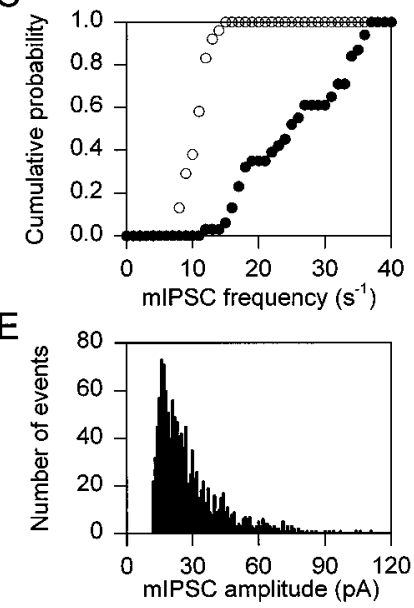

$\mathrm{F}$

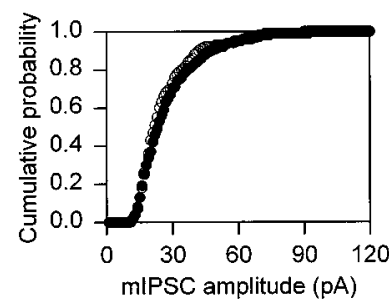

Figure 2. Astrocyte stimulation increases mIPSC frequency. $A$, mIPSCs recorded at a holding potential of $-30 \mathrm{mV}$ before and after mechanical stimulation of an astrocyte. $B$, Time course of the mIPSC frequency calculated in $1 \mathrm{sec}$ bins. Zero time corresponds to the time of astrocyte stimulation. $C$, Cumulative probability plot of the mIPSC frequency 30 sec before and after astrocyte stimulation (open and filled symbols, respectively). $D, E$, Histograms of mIPSC amplitudes recorded $30 \mathrm{sec}$ before and after astrocyte stimulation, respectively. $F$, Cumulative probability plot of the mIPSC amplitudes recorded $30 \mathrm{sec}$ before and after astrocyte stimulation (open and filled symbols, respectively). G, Average $(n=12)$ cumulative probability plot of the mEPSC amplitudes recorded $30 \mathrm{sec}$ before and after astrocyte stimulation (open and filled symbols, respectively). Cumulative probability plots were obtained as in Figure 1.

the ability of astrocyte stimulation to increase the frequency of mPSCs was reduced significantly (mEPSCs: $61.1 \pm 3.5 \%$ in control and $3.3 \pm 3.3 \%$ in thapsigargin; $p<0.001$; mIPSCs: $47.9 \pm 9.5 \%$ in control and $8.9 \pm 5.0 \%$ in thapsigargin; $p<0.01$ ), without altering their baseline frequency (the resting mean mEPSC frequency in control was $2.98 \pm 1.86 / \mathrm{sec}$ and was $3.30 \pm$ $1.24 / \mathrm{sec}$ in thapsigargin-treated cells; $n=8$ ). Although we cannot exclude the possibility that thapsigargin acted via neuronal $\mathrm{Ca}^{2+}$ stores, these results suggest that a $\mathrm{Ca}^{2+}$ elevation in astrocytes is required for the stimulus-induced increase in $\mathrm{mPSC}$ frequency.

To control further for nonspecific effects of stimulation and to remove any lingering concern regarding direct activation of neuronal processes, we specifically blocked $\mathrm{Ca}^{2+}$ elevations in astrocytes by microinjecting the $\mathrm{Ca}^{2+}$ chelator BAPTA into individual astrocytes. BAPTA was injected into a single astrocyte along with the fluorescent indicator fluoro-ruby (a dextran-conjugated 

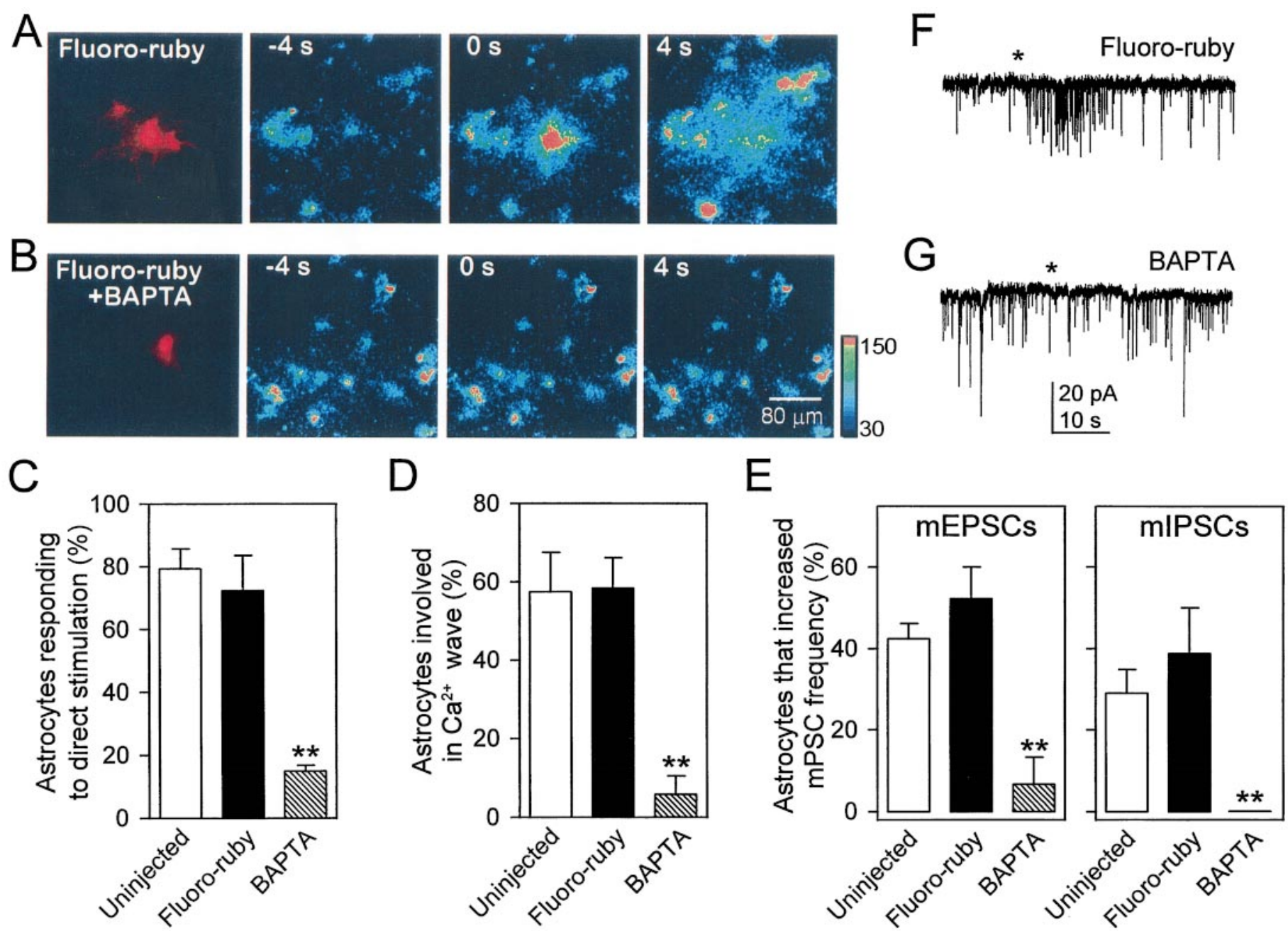

Figure 3. Microinjection of the $\mathrm{Ca}^{2+}$ chelator BAPTA into an astrocyte prevents the propagation of astrocyte $\mathrm{Ca}^{2+}$ waves and blocks the astrocyte-induced increase in mPSC frequency. $A$, Cultures were loaded with the $\mathrm{Ca}^{2+}$ indicator fluo- 3 to monitor the stimulus-induced $\mathrm{Ca}^{2+}$ elevations in astrocytes, and a single astrocyte was microinjected with fluoro-ruby (left panel). Right panels show images in pseudocolor mode representing intensity of fluo-3 emission taken before, during, and after mechanical stimulation of the fluoro-ruby-injected cell at the times indicated. Zero time corresponds to the time of astrocyte stimulation. Mechanical stimulation increases intracellular $\mathrm{Ca}^{2+}$ in the injected cell as well as in neighboring unstimulated astrocytes. $B$, Same as in $A$ but with a single astrocyte microinjected with fluoro-ruby and BAPTA (left panel). Mechanical stimulation of the injected cell did not change the fluorescent emission of fluo-3 either in the stimulated or neighboring astrocytes. $C, D$, Quantitative data taken from these experiments. The number of astrocytes involved in $\mathrm{Ca}^{2+}$ waves was quantified by the proportion of nonstimulated cells within the field of view that responded with a $\mathrm{Ca}^{2+}$ elevation. Although the ability of astrocytes to respond to direct stimulation or to evoke $\mathrm{Ca}^{2+}$ waves $(C$ and $D$, respectively) was unaffected by the injection of fluoro-ruby, it was reduced significantly by BAPTA injection. In parallel studies mPSCs were recorded in response to mechanical stimulation of astrocytes. Whereas the astrocyte-induced mPSC frequency increase was not affected by the injection of fluoro-ruby $(E, F)$, it was prevented by the injection of BAPTA $(E, G) .{ }^{* *} p<0.01$.

dye that does not pass through gap junctions, thus labeling only the injected cell) (Fig. 3A,B, left panels), and cells were allowed to recover for $1 \mathrm{hr}$ before the onset of a recording experiment. During this time the cells were loaded with the $\mathrm{Ca}^{2+}$ indicator fluo-3 so that we could confirm optically that BAPTA injection blocked the stimulus-induced $\mathrm{Ca}^{2+}$ waves. Stimulation of an uninjected astrocyte reliably evoked (18 of 22) an increase in its intracellular $\mathrm{Ca}^{2+}$ that resulted in $\mathrm{Ca}^{2+}$ increments in adjacent astrocytes. Direct stimulation of cells that had been injected with fluoro-ruby alone demonstrated that the injection protocol did not affect $\mathrm{Ca}^{2+}$ wave generation, because it did not change the ability of astrocytes to respond to direct stimulation nor to evoke $\mathrm{Ca}^{2+}$ waves (Fig. 3A,C,D). However, when astrocytes injected with BAPTA were stimulated, there was a significant reduction in the proportion that responded with a $\mathrm{Ca}^{2+}$ elevation as well as a reduced proportion of cells involved in the $\mathrm{Ca}^{2+}$ wave (Fig. $3 B-D)$. Thus, microinjection of BAPTA, and not the injection procedure per se, significantly reduced $\mathrm{Ca}^{2+}$ wave generation in astrocytes.

To determine whether a $\mathrm{Ca}^{2+}$ wave in the astrocyte was necessary for the stimulus-induced increase in frequency of mPSCs we performed similar microinjection experiments before recording whole-cell currents from neurons. After microinjection, a fluoro-ruby-containing astrocyte was brought in the field of view, and mPSCs were recorded from an adjacent neuron that was located within $\sim 150 \mu \mathrm{m}$ of the injected astrocyte. Microinjection of fluoro-ruby alone did not impair the astrocyte-induced increase in frequency of mPSCs. However, when astrocytes were injected with BAPTA, we observed a significant reduction in the ability of astrocyte stimulation to increase the frequency of 


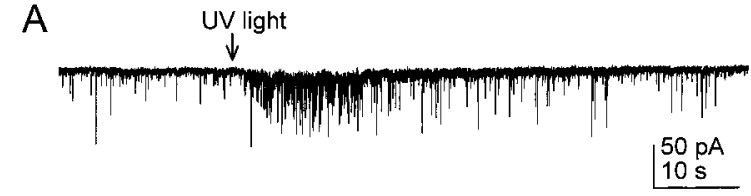

$\mathrm{B}$
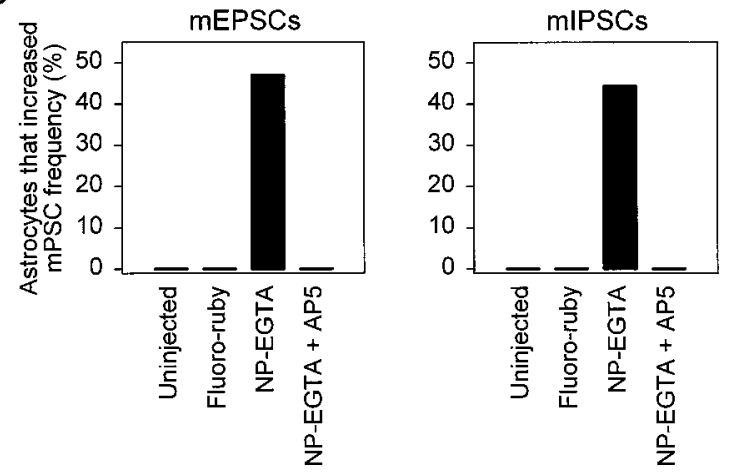

Figure 4. $\mathrm{Ca}^{2+}$ elevation in astrocytes is sufficient to increase the frequency of mPSCs. $A$, Whole-cell recording from a neuron adjacent to an astrocyte that had been microinjected with the $\mathrm{UV}$-sensitive $\mathrm{Ca}^{2+}$ cage NP-EGTA. UV photolysis (arrow) increased the $\mathrm{Ca}^{2+}$ level in the astrocyte and caused an increase in the frequency of mEPSCs. B, Graphs summarizing the effects of photolysis on the mEPSC and mIPSC frequency. Pulses of UV light only increased the frequency of mPSCS when the astrocyte was injected with NP-EGTA. Although the frequency of mPSCs was not modified by UV stimulation of uninjected astrocytes ( 0 of 6 cells) or astrocytes injected with fluoro-ruby alone ( 0 of 22 cells), photolysis of NP-EGTA-injected astrocytes increased the frequency of mEPSCs ( 8 of 17 cells) and mIPSCs ( 4 of 9 cells). This photolysisdependent increase in mPSC frequency was prevented by incubation with $50 \mu \mathrm{M}$ AP5 (0 of 12 NP-EGTA-injected astrocytes).

mPSCs (Fig. 3E-G). Because this inhibitory action is specific to the presence of BAPTA and not to injection per se, we can conclude that it is a result of the chelation of $\mathrm{Ca}^{2+}$ rather than a result of nonspecific effects of injection. Taken together with data obtained from experiments with C-FITC tetanus toxin labeling and with thapsigargin treatment, these BAPTA microinjection experiments demonstrate that a $\mathrm{Ca}^{2+}$ elevation, specifically in astrocytes, as opposed to an inadvertent stimulation of neuronal processes, is necessary for the stimulus-induced increase in mPSC frequency.

To investigate further the role of $\mathrm{Ca}^{2+}$ in the astrocyteinduced modulation of synaptic transmission, we asked whether a $\mathrm{Ca}^{2+}$ elevation in astrocytes was sufficient to increase the frequency of mPSCs. Single astrocytes were microinjected with the UV-sensitive $\mathrm{Ca}^{2+}$ cage NP-EGTA (together with the fluorescent dye fluoro-ruby to identify the injected cell), which was photolyzed by using one to six 3-nsec-UV (337 nm) pulses delivered through a UV-transmitting optical fiber (beam diameter, 10-15 $\mu \mathrm{m}$ ) (V. Parpura and P. Haydon, unpublished data). Photolysis reliably increased intracellular $\mathrm{Ca}^{2+}$ as monitored by using fluo-3 ( 4 of 4 cells). In parallel experiments analyzing the effects on neuronal currents, photolysis reliably evoked the previously characterized nonsynaptic slow inward current (Araque et al., 1998) as well as an increase in the frequency of mPSCs (Fig. $4 A, B)$. In contrast, UV stimulation of either the uninjected astrocytes or fluoro-ruby-injected astrocytes did not change the mPSC frequency (Fig. 4B). Taken together, these experiments demonstrate that an elevation of astrocyte $\mathrm{Ca}^{2+}$ is both neces- sary and sufficient to induce an increase in the frequency of both inhibitory and excitatory miniature synaptic currents.

\section{Astrocyte-induced synaptic enhancement is mediated by activation of NMDA receptors}

Several studies have demonstrated that an elevation of internal $\mathrm{Ca}^{2+}$ in astrocytes induces a $\mathrm{Ca}^{2+}$-dependent release of glutamate that can be sensed by adjacent neurons (Charles, 1994; Parpura et al., 1994; Hassinger et al., 1995; Pasti et al., 1997; Araque et al., 1998; Bezzi et al., 1998). We used pharmacological tools to determine the glutamate receptor dependence of the astrocyte-induced increase in mPSC frequency (Fig. 5). The incidence of the astrocyte-mediated increase of mIPSC or mEPSC frequency was unaffected by the mGluR antagonists $(S)$-2-amino-2-methyl-4-phosphonobutanoic acid (MAP4; 0.5 $\mathrm{mM}$ ) or $(S)$ - $\alpha$-methyl-4-carboxyphenylglycine (MCPG; $0.5 \mathrm{~mm}$ ). The non-NMDA glutamate receptor antagonist 6-cyano-7nitroquinoxaline-2,3-dione (CNQX, $10 \mu \mathrm{M}$; because mEPSCs are sensitive to this antagonist, the effect of CNQX on miniature frequency was assayed only on mIPSCs) did not reduce the ability of astrocytes to induce an increase in mIPSC frequency. However, either the presence of $4 \mathrm{mM} \mathrm{Mg}^{2+}$ or of the NMDA glutamate receptor antagonist AP5 $(50 \mu \mathrm{M})$ dramatically reduced the astrocyte-induced increase in MIPSC and mEPSC frequency. To test further the role of NMDA receptors in mediating the modulation of mPSC frequency, we incubated cultures for $5 \mathrm{~min}$ in NMDA $(200 \mu \mathrm{M})$ and MK-801 (5 $\mu \mathrm{M}$; an irreversible open channel blocker of NMDA receptors) to cause a sustained block of this receptor subtype. After washout, astrocyte stimulation no longer modulated mPSC frequency (Fig. 5). Additionally, in photolysis experiments, AP5 blocked the ability of photolytic $\mathrm{Ca}^{2+}$ elevations in astrocytes to raise mPSC frequency (see Fig. 4B). These data demonstrate that the enhancement of the miniature synaptic frequency was mediated selectively by the activation of NMDA receptors.

\section{Astrocyte-induced increase in MPSC frequency is not mediated by synaptically located NMDA receptors}

To evaluate the location of NMDA receptors mediating the astrocyte-dependent modulation of mPSC frequency, we selectively blocked the synaptically located NMDA receptors with MK-801, an irreversible use-dependent open channel blocker of NMDA receptors (Hessler et al., 1993; Rosenmund et al., 1993; Reid et al., 1997), and asked whether the AP5-sensitive astrocyte modulation of mPSC frequency persisted (Fig. 6A). To achieve this block of synaptic NMDA receptors, we applied MK-801 (5 $\mu \mathrm{M}$ ) while activating synapses with high osmolarity solution (1- to 50 -sec-duration pressure pulses of solution with $0.3 \mathrm{M}$ sucrose added) (Fig. 6A). Subsequently, MK-801 was washed out of the bathing saline. Although the effect of hyperosmotic saline on astrocytes is not well defined, it previously has been documented that this maneuver reliably evokes quantal transmitter release at synapses (Fatt and Katz, 1952; Malgaroli and Tsien, 1992; Manabe et al., 1992). To determine whether we successfully blocked synaptically located NMDA receptors, we analyzed the decay kinetics of mEPSCs. Previous studies have shown that EPSCs decay with biexponential kinetics that reflect two components: a fast CNQX- and a slow AP5-sensitive component (Bekkers and Stevens, 1989; McBain and Dingledine, 1992). We first confirmed in control conditions that mEPSCs showed a fast $(\tau=$ $5.6 \pm 0.8 \mathrm{msec})$ and a slow $(\tau=45.1 \pm 11.1 \mathrm{msec})$ component $(n=6)$ (Fig. 6B, Control) and that the latter was abolished by 50 

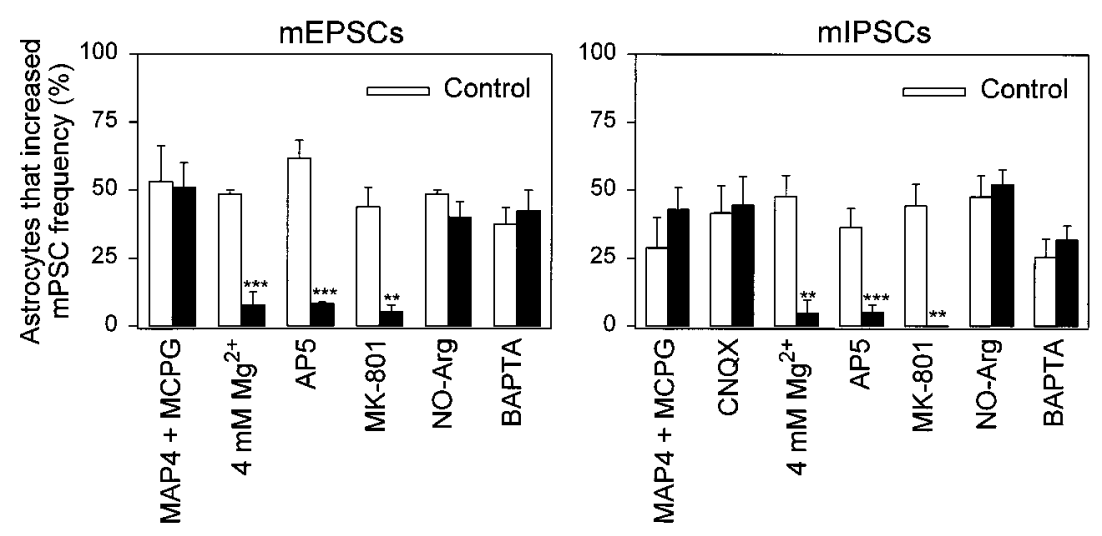

A

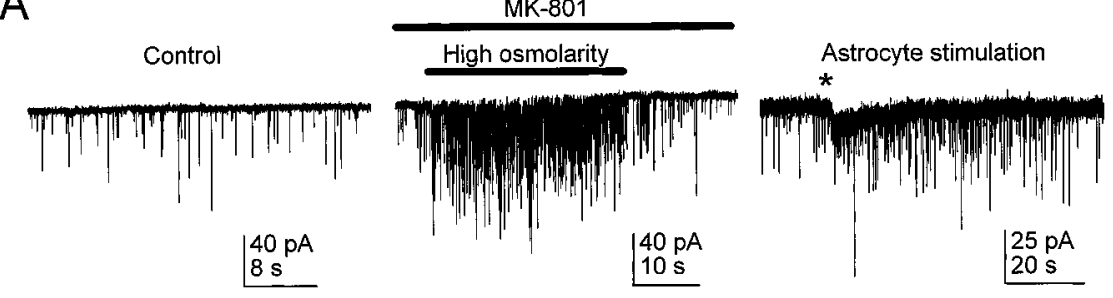

B

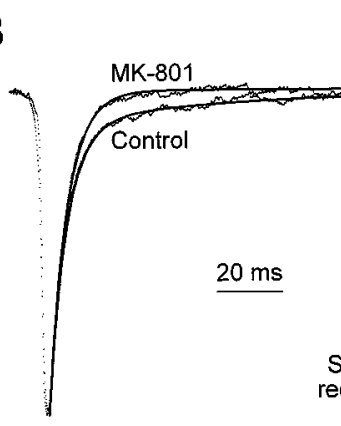

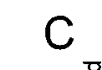

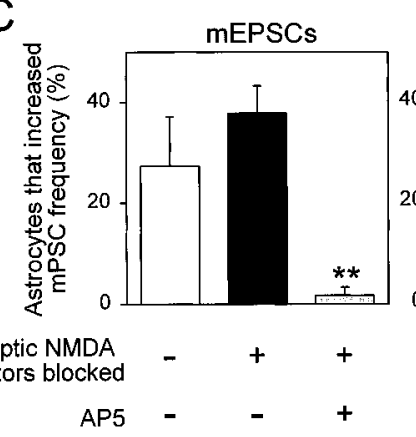

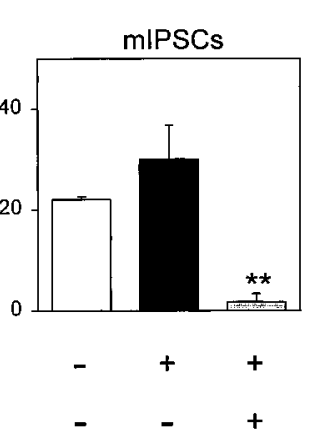

Figure 5. Astrocyte-induced increase in MPSC frequency is mediated by NMDA receptors. Shown is the percentage of mechanically stimulated astrocytes that increased the frequency of mEPSCs (left) and mIPSCs (right) in $0.5 \mathrm{mM}$ MAP4 and $0.5 \mathrm{mM} \mathrm{MCPG}, 10 \mu \mathrm{M}$ CNQX, $4 \mathrm{~mm} \mathrm{Mg}^{2+}$, $50 \mu \mathrm{M}$ AP5, and $50 \mu \mathrm{M}$ NO-Arg; also shown is the percentage after dialysis of the postsynaptic cell with BAPTA (10 mM in the recording pipette) and in their respective control solution in parallel cultures. In the histograms labeled MK-801 we caused a use-dependent block of NMDA receptors before stimulating astrocytes by incubating the cultures during 5 min in NMDA $(200 \mu \mathrm{M})$ and MK-801 $(5 \mu \mathrm{M})$ to cause a sustained block of this receptor subtype (see Results). After washout, astrocyte stimulation no longer modulated mPSC frequency. Significant differences with respect to control were established by the Student's $t$ test at ${ }^{* *} p<0.01 ; * * p<0.001$.

Figure 6. AP5-sensitive astrocyte-induced increase in $\mathrm{mPSC}$ frequency is not mediated by synaptic NMDA receptors. $A$, mPSCs recorded at a holding potential of $-60 \mathrm{mV}$ in control solution (left). To block postsynaptic NMDA receptors, we added MK801 to the saline while the synaptic release of glutamate was stimulated by the pressure ejection of high osmolarity saline (center). Several 1- to 50-secduration pressure pulses of high osmolarity solution (obtained by the addition of $0.3 \mathrm{M}$ sucrose to the standard saline) were delivered, but only one is shown. Subsequently, MK-801 was washed out of the saline. Right, mPSCs recorded at a holding potential of $-30 \mathrm{mV}$ after the blockage of postsynaptic NMDA receptors with MK-801. The trace has been offset for illustration purposes. Mechanical stimulation of the astrocyte is indicated by the asterisk. Note that, despite the selective block of synaptic NMDA receptors, the stimulation of astrocytes still evoked an increase in the frequency of mPSCs. $B$, Averaged $(n>50)$ mEPSCs (dotted lines) in control solution and in MK-801 after several pressure pulses of high osmolarity solution. Synaptic activation with high osmolarity saline blocked postsynaptic NMDA receptors, because mEPSCs now exhibited only one time constant of decay. The decay time course of mEPSCs was fit to two and to a single exponential function in control and after MK-801 treatment, respectively (continuous lines). C, Proportion of astrocytes in which mechanical stimulation evoked an increase in the frequency of mEPSCs (left) and mIPSCs (right) in control solution and after the blockage of synaptic NMDA receptors with MK-801 and high osmolarity saline in the absence and in the presence of $50 \mu \mathrm{M} \mathrm{AP5.}{ }^{* *} p<0.01$.

$\mu \mathrm{M}$ AP5, whereas the fast component remained unchanged $(\tau=$ $6.6 \pm 0.8 \mathrm{msec}$ ) (data not shown). After treatment of the synapses with MK-801 and hyperosmotic solution, the decay phase of the mEPSCs could be fit by a single exponential with a time constant $(6.6 \pm 1.6 \mathrm{msec})$ that was not significantly different from the fast time constant in control (Fig. 6B, $M K-801$ ), confirming that postsynaptic NMDA receptors were blocked. Additional evidence that MK-801 had blocked the postsynaptic NMDA receptors was provided by the inability of AP5 to modify further the mEPSC decay time constant $(6.0 \pm 0.6$ and $6.4 \pm 0.7 \mathrm{msec}$ before and after AP5, respectively; $n=4)$.

Once the block of synaptic NMDA receptors was confirmed, we determined whether astrocytes could increase the frequency of mPSCs in the absence of MK-801 in the bathing saline. Despite the sustained open channel blockade of synaptic NMDA receptors by MK-801, the stimulation of astrocytes still induced an increase in mPSC frequency that was sensitive to AP5 (Fig. $6 A, C)$. Because synaptic NMDA receptors were blocked by MK801, yet astrocytes still evoked an AP5-sensitive increase in mPSC frequency, we conclude that extrasynaptically located NMDA receptors mediate this form of synaptic modulation.
To evaluate further the role of the postsynaptic NMDA receptors in mediating the astrocyte-induced increase in mPSC frequency, we dialyzed the $\mathrm{Ca}^{2+}$ chelator BAPTA $(10 \mathrm{~mm})$ in the postsynaptic cell and incubated cells with NO-Arg $(50 \mu \mathrm{M})$ that inhibits the production of nitric oxide, a candidate retrograde messenger putatively involved in some forms of synaptic plasticity (Schuman and Madison, 1991; Haley et al., 1992). Neither of these manipulations affected the ability of astrocytes to modulate the frequency of mPSCs (see Fig. 5), suggesting that changes in the postsynaptic neuron and specifically in its $\left[\mathrm{Ca}^{2+}\right]_{i}$ are not necessary to trigger the astrocyte-induced synaptic modulation.

\section{DISCUSSION}

Recent experimental evidence presented by several laboratories has challenged the traditional idea of astrocytes as being supporting cells for neurons and has suggested a more active role for astrocytes in the nervous system. It has been shown that astrocytes exhibit a form of excitability on the basis of intracellular $\mathrm{Ca}^{2+}$ variations and that they can communicate among themselves via propagating intercellular $\mathrm{Ca}^{2+}$ waves (Cornell-Bell et al., 1990; Charles et al., 1991; Finkbeiner, 1992; Newman and 
Zahs, 1997). Moreover, astrocytes, which express several neurotransmitter receptors (Cornell-Bell et al., 1990; Charles et al., 1991; Murphy et al., 1993; Duffy and MacVicar, 1995), may respond to neuronal activity by generating $\mathrm{Ca}^{2+}$ waves (Dani et al., 1992; Porter and MacCarthy, 1996), indicating that neurons can signal to astrocytes. Furthermore, astrocytes also may signal to neurons, because astrocyte stimulation can induce $\mathrm{Ca}^{2+}$ elevations in neurons (Charles, 1994; Nedergaard, 1994; Parpura et al., 1994; Hassinger et al., 1995; Pasti et al., 1997; Araque et al., 1998; Bezzi et al., 1998). Therefore, these results suggest that neurons and astrocytes may function as an interdependent network with bidirectional communication between these elements.

However, despite the recent accumulating data describing the existence as well as the mechanisms of astrocyte-neuron signaling, very little is known about their physiological implications. In a first report addressing that question, we determined the consequences of astrocyte stimulation on electrophysiological properties and on action potential-evoked synaptic transmission of hippocampal neurons (Araque et al., 1998). We demonstrated that astrocytes can evoke glutamate-dependent, NMDA, and nonNMDA receptor-mediated slow inward currents in neurons. We also have shown that astrocytes can modulate action potentialevoked synaptic transmission by the activation of presynaptic mGluRs. In the present study we show additional physiological consequences of astrocyte stimulation on neuronal function. Indeed, the results presented above show that astrocyte stimulation may increase the frequency of both mEPSCs and mIPSCs. One concern, however, is whether our stimuli, which we direct to the astrocyte, do elevate the $\mathrm{Ca}^{2+}$ level specifically only in the astrocyte. Experiments that used thapsigargin, intracellular BAPTA injection into single astrocytes, and single-cell UV photolysis of NP-EGTA support previous findings indicating that an intracellular $\mathrm{Ca}^{2+}$ elevation in astrocytes is both necessary and sufficient to evoke glutamate release and thus the glutamate receptor-dependent neuronal responses.

Classically, it is considered that changes in the frequency of mPSCs reflect a modification in the probability of the presynaptic transmitter release (see Del Castillo and Katz, 1954; Malgaroli and Tsien, 1992; Manabe et al., 1992; Wyllie et al., 1994). We have shown that astrocyte stimulation increased the frequency of $\mathrm{mP}$ SCs without modifying their amplitude distribution, suggesting that astrocytes induced an alteration of the presynaptic terminal, increasing the probability of transmitter release. The simplest interpretation of our present results would be that the effects of astrocyte stimulation are mediated by NMDA receptors located in the presynaptic terminal. However, they could not be located in the synaptic junction per se, because experiments in which we caused a use-dependent block of synaptic NMDA receptors demonstrate that glutamate released from astrocytes accesses NMDA receptors located extrasynaptically. Although the presence of NMDA receptors in presynaptic terminals of the CNS remains to be determined, such a localization has been postulated by numerous studies that used immunocytochemical localization (Aoki et al., 1994; Liu et al., 1994; Siegel et al., 1994; Johnson et al., 1996; Van Bockstaele and Colago, 1996) and biochemical analysis of neurotransmitter release (Fink et al., 1990; Krebs et al., 1991; Martin et al., 1991; Desce et al., 1992; Pittaluga and Raiteri, 1992; Cheramy et al., 1996; Wang and Thukral, 1996). However, further studies will be required to pinpoint the location of the NMDA receptors responsible for the astrocyte-induced modulation of the synapse.

We have demonstrated previously that astrocyte stimulation reduced the magnitude of action potential-evoked excitatory and inhibitory synaptic currents by decreasing the probability of evoked transmitter release via the activation of presynaptic mGluRs (Araque et al., 1998). This result seems to contrast with the present data showing an increase in the frequency of mPSCs. However, these data can be explained because both phenomena are mediated by separate mechanisms. Indeed, evoked, but not spontaneous, transmitter release depends on $\mathrm{Ca}^{2+}$ influx through presynaptic $\mathrm{Ca}^{2+}$ channels that can be inhibited by presynaptic mGluRs (Takahashi et al., 1996). Thus, the modulation of evoked synaptic transmission is not necessarily associated with changes in the frequency of spontaneous synaptic transmission (Araque et al., 1994; Gereau and Conn, 1995; Sciancalepore et al., 1995; Fitzsimonds and Dichter, 1996).

In our experiments we optimized the ability of astrocytes to evoke the NMDA receptor-dependent increase in mPSC frequency by removing $\mathrm{Mg}^{2+}$ from the extracellular saline. However, under normal extracellular $\mathrm{Mg}^{2+}$ concentration (2 $\mathrm{mm}$ ) astrocytes still can induce this response, albeit with a lower incidence $\left(50.7 \pm 5.6 \%\right.$ in $0 \mathrm{mM} \mathrm{Mg}^{2+}$ as compared with $18.7 \pm$ $10.9 \%$ in $2 \mathrm{~mm} \mathrm{Mg}^{2+}$ ) (see also Araque et al., 1998). Although this demonstrates that astrocytes can cause NMDA receptordependent increases in mPSC frequency under normal extracellular conditions, it also suggests that this pathway is likely to be activated most effectively under conditions that facilitate ion conduction through this channel, such as during neuronal depolarization that occurs during the induction of long-term potentiation. Thus, one might envision that the astrocyte-induced mGluR-dependent depression of evoked transmission might give way to NMDA receptor-dependent facilitation of synapses under depolarizing conditions.

In conclusion, we have provided evidence that demonstrates in cell culture that an elevation of $\mathrm{Ca}^{2+}$ in astrocytes is both necessary and sufficient to increase the mPSC frequency of adjacent neurons. This astrocyte-induced enhancement of synaptic transmission is AP5-sensitive but is not mediated by NMDA receptors that have access to neurotransmitter released at synapses, as indicated by experiments that used the open channel blocker of NMDA receptors, MK-801, together with synaptic activation of these receptors. Consequently, we conclude that a $\mathrm{Ca}^{2+}$ elevation in astrocytes induces the release of the transmitter glutamate, which causes an NMDA receptor-dependent increase in mPSC frequency by acting on extrasynaptic NMDA receptors, which in turn increases the probability of transmitter release from the presynaptic terminal. Our data reveal new physiological consequences of the proposed existence of bidirectional communication between neurons and astrocytes (Charles, 1994; Nedergaard, 1994; Parpura et al., 1994; Hassinger et al., 1995; Pasti et al., 1997; Araque et al., 1998; Bezzi et al., 1998) and support a more active role of astrocytes in information processing in the brain and potentially in supporting synaptic plasticity.

\section{REFERENCES}

Aoki C, Venkatesan C, Go CG, Mong JA, Dawson TM (1994) Cellular and subcellular localization of NMDA-R1 subunit immunoreactivity in the visual cortex of adult and neonatal rats. J Neurosci 14:5202-5222.

Araque A, Clarac F, Buño W (1994) P-type $\mathrm{Ca}^{2+}$ channels mediate excitatory and inhibitory synaptic transmitter release in crayfish muscle. Proc Natl Acad Sci USA 91:4224-4228.

Araque A, Parpura V, Sanzgiri RP, Haydon PG (1998) Glutamatedependent astrocyte modulation of synaptic transmission between cultured hippocampal neurons. Eur J Neurosci 10:2129-2142.

Bekkers JM, Stevens CF (1989) NMDA and non-NMDA receptors are 
colocalized at individual excitatory synapses in cultured rat hippocampus. Nature 341:230-233.

Bezzi P, Carmignoto G, Pasti L, Vesce S, Rossi D, Lodi Rizzini B, Pozzan T, Volterra A (1998) Prostaglandins stimulate calciumdependent glutamate release in astrocytes. Nature 391:281-285.

Charles AC (1994) Glia-neuron intercellular calcium signaling. Dev Neurosci 16:196-206.

Charles AC, Merrill JE, Dirksen ER, Sanderson MJ (1991) Intercellular signaling in glial cells: calcium waves and oscillations in response to mechanical stimulation and glutamate. Neuron 6:983-992.

Charles AC, Dirksen ER, Merrill JE, Sanderson MJ (1993) Mechanisms of intercellular calcium signaling in glial cells studied with dantrolene and thapsigargin. Glia 7:134-145.

Cheramy A, Godeheu G, L'Hirondel M, Glowinski J (1996) Cooperative contributions of cholinergic and NMDA receptors in the presynaptic control of dopamine release from synaptosomes of the rat striatum. J Pharmacol Exp Ther 276:616-625.

Cornell-Bell AH, Finkbeiner SM, Cooper MS, Smith SJ (1990) Glutamate induces calcium waves in cultured astrocytes: long-range glial signaling. Science 247:470-473.

Dani JW, Chernjavsky A, Smith SJ (1992) Neuronal activity triggers calcium waves in hippocampal astrocyte networks. Neuron 8:429-440.

Del Castillo J, Katz B (1954) Quantal components of the endplate potential. J Physiol (Lond) 124:560-573.

Desce JM, Godeheu G, Galli T, Artaud F, Cheramy A, Glowinski J (1992) L-Glutamate-evoked release of dopamine from synaptosomes of the rat striatum: involvement of AMPA and NMDA receptors. Neuroscience 47:333-339.

Duffy S, MacVicar BA (1995) Adrenergic calcium signaling in astrocyte networks within the hippocampal slice. J Neurosci 15:5535-5550.

Fatt P, Katz B (1952) Spontaneous subthreshold activity at motor nerve endings. J Physiol (Lond) 117:109-128.

Fink K, Bonish H, Gothert M (1990) Presynaptic NMDA receptors stimulate noradrenaline release in the cerebral cortex. Eur J Pharmacol 185:115-117.

Finkbeiner SM (1992) Calcium waves in astrocytes-filling in the gaps. Neuron 8:1101-1108.

Fitzsimonds RM, Dichter MA (1996) Heterologous modulation of inhibitory synaptic transmission by metabotropic glutamate receptors in cultured hippocampal neurons. J Neurophysiol 75:885-893.

Gereau RW, Conn PJ (1995) Multiple presynaptic metabotropic glutamate receptors modulate excitatory and inhibitory synaptic transmission in hippocampal area CA1. J Neurosci 15:6879-6889.

Haley JE, Wilcox GL, Chapman PF (1992) The role of nitric oxide in hippocampal long-term potentiation. Neuron 8:211-216.

Hassinger TD, Atkinson PB, Strecker GJ, Whalen LR, Dudek FE, Koseel AH, Kater SB (1995) Evidence for glutamate-mediated activation of hippocampal neurons by glial calcium waves. J Neurobiol 28:159-170.

Hessler NA, Shirke AM, Malinow R (1993) The probability of transmitter release at a mammalian central synapse. Nature 366:569-572.

Johnson RR, Jiang X, Burkhalter A (1996) Regional and laminar differences in synaptic localization of NMDA receptor subunit NR1 splice variants in rat visual cortex and hippocampus. J Comp Neurol 368:335-355.

Krebs MO, Trovero F, Desban M, Gauchy C, Glowinski J, Kemel ML (1991) Distinct presynaptic regulation of dopamine release through NMDA receptors in striosome- and matrix-enriched areas of the striatum. J Neurosci 11:1256-1262.

Liu H, Wang H, Sheng M, Jan LY, Jan YN, Basbaum AI (1994) Evidence for presynaptic $N$-methyl-D-aspartate autoreceptors in the spinal cord dorsal horn. Proc Natl Acad Sci USA 91:8383-8387.

Malgaroli A, Tsien RW (1992) Glutamate-induced long-term potentiation of the frequency of miniature synaptic currents in cultured hippocampal neurons. Nature 357:134-139.
Manabe T, Renner P, Nicoll RA (1992) Postsynaptic contribution to long-term potentiation revealed by the analysis of miniature synaptic currents. Nature 355:50-55.

Martin D, Bustos GA, Bowe MA, Brady SD, Nadler JV (1991) Autoreceptor regulation of glutamate and aspartate release from slices of the hippocampal CA1 area. J Neurochem 56:1647-1655.

McBain CJ, Dingledine R (1992) Dual-component miniature excitatory synaptic currents in rat hippocampal CA3 pyramidal neurons. J Neurophysiol 68:16-27.

Murphy TH, Blatter LA, Wier WG, Baraban JM (1993) Rapid communication between neurons and astrocytes in primary cortical cultures. J Neurosci 13:2672-2679.

Nedergaard M (1994) Direct signaling from astrocytes to neurons in cultures of mammalian brain cells. Science 263:1768-1771.

Newman EA, Zahs KR (1997) Calcium waves in retinal glial cells. Science 275:844-847.

Parpura V, Basarsky TA, Liu F, Jeftinija K, Jeftinija S, Haydon PG (1994) Glutamate-mediated astrocyte-neuron signaling. Nature 369:744-747.

Pasti L, Volterra A, Pozzan T, Carmignoto G (1997) Intracellular calcium oscillations in astrocytes: a highly plastic, bidirectional form of communication between neurons and astrocytes in situ. J Neurosci 17:7817-7830.

Pittaluga A, Raiteri M (1992) $N$-methyl-D-aspartic acid (NMDA) and non-NMDA receptors regulating hippocampal norepinephrine release. I. Localization on axon terminals and pharmacological characterization. J Pharmacol Exp Ther 260:232-237.

Porter JT, McCarthy KD (1996) Hippocampal astrocytes in situ respond to glutamate released from synaptic terminals. J Neurosci 16:5073-5081.

Reid CA, Clements JD, Bekkers JM (1997) Nonuniform distribution of $\mathrm{Ca}^{2+}$ channel subtypes on presynaptic terminals of excitatory synapses in hippocampal cultures. J Neurosci 17:2738-3745.

Rosenmund C, Clements JD, Westbrook GL (1993) Nonuniform probability of glutamate release at a hippocampal synapse. Science 262:754-757.

Schuman EM, Madison DV (1991) A requirement for the intercellular messenger nitric oxide in long-term potentiation. Science 254:1503-1506.

Sciancalepore M, Stratta F, Fisher ND, Cherubini E (1995) Activation of metabotropic glutamate receptors increase the frequency of spontaneous GABAergic currents through protein kinase $\mathrm{A}$ in neonatal rat hippocampal neurons. J Neurophysiol 74:1118-1122.

Siegel SJ, Brose N, Janssen WG, Gasic GP, Jahn R, Heinemann SF (1994) Regional, cellular, and ultrastructural distribution of $N$-methylD-aspartate receptor subunit 1 in monkey hippocampus. Proc Natl Acad Sci USA 91:564-568.

Takahashi T, Forsythe ID, Tsujimoto T, Barnes-Davies M, Onodera K (1996) Presynaptic calcium current modulation by a metabotropic glutamate receptor. Science 274:594-597.

Van Bockstaele EJ, Colago EE (1996) Selective distribution of the NMDA-R1 glutamate receptor in astrocytes and presynaptic axons terminals in the nucleus locus coeruleus of the rat brain: an immunoelectron microscopic study. J Comp Neurol 369:483-496.

Wang JKT, Thukral V (1996) Presynaptic NMDA receptors display physiological characteristics of homomeric complexes of NR1 subunits that contain the exon 5 insert in the $\mathrm{N}$-terminal domain. J Neurochem 66:865-868.

Wyllie DA, Manabe T, Nicoll RA (1994) A rise in postsynaptic $\mathrm{Ca}^{2+}$ potentiates miniature excitatory postsynaptic currents and AMPA responses in hippocampal neurons. Neuron 12:127-138. 